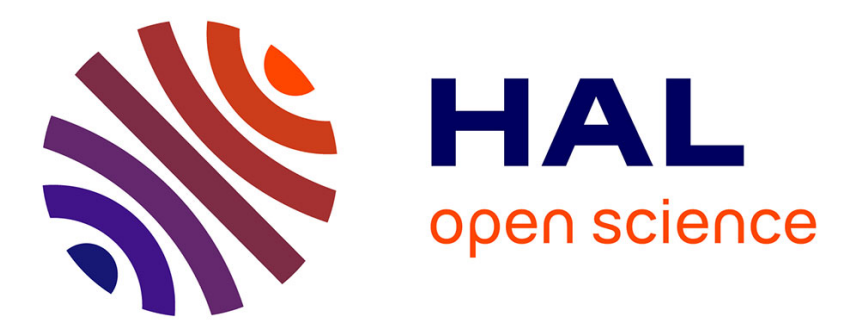

\title{
A New Strategy for Automatic Coupling Between the Inductive PEEC Method and an Integral Electrostatic Formulation
}

Quang-Anh Phan, Gerard Meunier, Olivier Chadebec, Jean-Michel Guichon, Bertrand Bannwarth

\section{To cite this version:}

Quang-Anh Phan, Gerard Meunier, Olivier Chadebec, Jean-Michel Guichon, Bertrand Bannwarth. A New Strategy for Automatic Coupling Between the Inductive PEEC Method and an Integral Electrostatic Formulation. IEEE Transactions on Electromagnetic Compatibility, 2022, 4 (2), pp.506-515. 10.1109/TEMC.2021.3114511 . hal-03381319

\section{HAL Id: hal-03381319 https://hal.science/hal-03381319}

Submitted on 16 Oct 2021

HAL is a multi-disciplinary open access archive for the deposit and dissemination of scientific research documents, whether they are published or not. The documents may come from teaching and research institutions in France or abroad, or from public or private research centers.
L'archive ouverte pluridisciplinaire HAL, est destinée au dépôt et à la diffusion de documents scientifiques de niveau recherche, publiés ou non, émanant des établissements d'enseignement et de recherche français ou étrangers, des laboratoires publics ou privés. 


\title{
A new strategy for automatic coupling between the inductive PEEC method and an integral electrostatic formulation
}

\author{
Quang-Anh Phan ${ }^{1}$, Gerard Meunier ${ }^{1}$, Olivier Chadebec ${ }^{1}$, Jean-Michel Guichon ${ }^{1}$, Bertrand Bannwarth ${ }^{1}$ \\ ${ }^{1}$ University Grenoble Alpes, CNRS, Grenoble INP, G2Elab, 38000 Grenoble, France
}

\begin{abstract}
This paper presents a new strategy for building an equivalent RLMC circuit for electromagnetic interconnections modelling in the context of the Partial Element Equivalent Circuit (PEEC) method. The method consists in splitting the overall formulation (RLMC) into two independent formulations (RLM) and (C). Both formulations are used independently to calculate equivalent circuit elements. Once all the elements are calculated, they are merged into a global electrical model which is solved globally with an external circuit solver. A coupling with the similar idea was presented a few years ago and showed very good performance for the modelling of electromagnetic devices where propagative effects are negligible like in power electronics applications. However, the coupling of the parasitic capacitances C with RLM circuit had to be carried out manually. Our new strategy allows to do it in a completely automatic way.
\end{abstract}

Index Terms-Partial Element Equivalent Circuit (PEEC), capacitance extraction, circuit coupling.

$\mathbf{T}$ HE PEEC method (Partial Element Equivalent Circuit) has been developed for many years. First introduced through studies of A. Ruehli [1] and then through a series of remarkable developments up to now [2], [3], [4], [5], [6], [7], [8], this method is today a very robust and powerful electromagnetic simulation tool which can be used for the modelling of a wide range of applications such as antennas, PCB, power electronic devices, shielding or others electromagnetic devices. More recent works have led to the development of the unstructured PEEC method allowing the use of unstructured general meshes thanks to face shape functions (Whitney 2form shape functions) [9] for the interpolation of current density. Starting from the work of F. Freschi [10] which was limited to conductive surfaces, extensions to volume regions have been proposed in [11] allowing simulations in a wide frequency range of problems with dielectric [12], [13] and magnetic materials [14], [15]. Today, PEEC is a very general computational method allowing the resolution of fullwave Maxwell's equations with few limiting assumptions. The formulation we proposed in [13], [12] is a very efficient one for the modelling of low to medium-frequency devices like power electronics devices. It includes resistive (R), inductive $(\mathrm{L}$ and $\mathrm{M})$ and also capacitive effects $(\mathrm{C})$ but neglects the propagation in media by lying on the Green's functions for the Laplace equation and not considering its Helmholtz's extension.The frequency range validity of this formulation is between $10 \mathrm{kHz}$ and $100 \mathrm{MHz}$ where the propagative effects can be neglected because the devices dimension we aim to study is about some tens of centimeters (wavelength at 200 $\mathrm{MHz}$ is $1.5 \mathrm{~m}$ ). However, in such problems, the skin effects have to be taken into account because the conductors have a width of tens of mm. Besides the capacitive effects can not be neglected because they generally appear from around $1 \mathrm{MHz}$ (depending on geometric dimensions of conductors) and are needed in the modelling of common mode current loop that develop between tracks and the ground planes.

In this formulation, all the elements of the mesh are associated to equivalent partial circuit elements. It leads to a global circuit with a huge number of components of very different natures and values so a poorly conditioned matrix system. This point is crucial in particular within the framework of matrix compression techniques [16], [17] where the convergence rate of the resolution is the main limitation of the method. An important research effort has been carried out on the subject. Much work has focused on the development of efficient preconditioning techniques in order to accelerate convergence [18], [17]. This topic of research is still very active because no "universal" preconditioner has yet been exhibited. This is why we have investigated others strategies in order to make PEEC method competitive to simulate problems with an industrial complexity. Few years ago, an alternative method has been proposed. It consists in splitting the global (RLMC) formulation in (RLM) and (C) independent formulations [19], [20], [21], [22]. Both formulations are used independently to compute equivalent circuit elements. Once all elements have been computed, they are merged in a global electric model which is solved in a global way thanks to an external circuit solver. An advantage of the approach is that both formulations do not need to be compatible meaning that different numerical techniques can be used to solve quasi-static (inductive) or electrostatic (capacitive) problems. For instance, in [19] and [20], the inductive problem has been solved with structured RLM-PEEC method while the equivalent capacitances have been computed with a boundary element method (BEM). This approach has led to matrix systems with smaller dimensions and good condition numbers and has shown its performance in terms of time and memory for the modelling of complex industrial power electronics devices. Even if the method has shown its efficiency, it remains difficult to use by a nonspecialist user. The main difficulty comes from the merge of both formulations and the building of the final circuit model. This task is complex and required a high expertise, the question being mainly the definition of the different capacitive regions and their connections in the global circuit to the inductive elements. To make this process automatic is absolutely necessary if we want the method to be used in 
an industrial context. This is what the paper aims to do by proposing a new algorithm based on an iterative resolution. The paper is organized as follows. The first part is dedicated to the presentation of the inductive RLM-PEEC formulation. The parasitic capacitances are computed with an equivalent charge integral formulation presented in part two. It is equivalent to a BEM approach. The third part of the paper is dedicated to the coupling strategy principle. It is based on the analysis of the electric potential distribution obtained after an initial resolution of the problem limited to the only inductive case. From the potential map obtained, a set of region is defined and used to compute with a BEM a set of capacitances which are introduced in a global circuit model. The resolution of these circuit equations leads to a new potential map which is analyzed once more in order to define a new set of regions associated with a new set of capacitances. Circuit equations are solved again and the process is repeated until convergence without any manual intervention of the user. The fourth part will give more details about the numerical implementation while the fifth will be dedicated to the validation of the method demonstrating its performance. The paper ends conclusions.

\section{UNSTRUCTURED INDUCTIVE RLM-PEEC}

Let us consider an electromagnetic quasi-static problem with linear, homogeneous and isotropic conductive regions. We have the Maxwell-Faraday equation:

$$
\nabla \times \mathbf{E}=-j \omega \mathbf{B}
$$

with $\omega$ the pulsation, $\mathbf{E}, \mathbf{B}$ the electric and magnetic fields respectively. Considering the free-divergence of $\mathrm{B}$, magnetic vector potential $\mathbf{A}$ and electric scalar potential $V$ are linked to $\mathrm{E}$ by

$$
\mathbf{E}=-j \omega \mathbf{A}-\nabla V
$$

For a problem without any magnetic material, the magnetic vector potential $\mathbf{A}$ is given by:

$$
\mathbf{A}=\frac{\mu_{0}}{4 \pi} \int_{\Omega_{c}} \frac{\mathbf{J}}{r} d \Omega_{c}
$$

with $\mathbf{J}$ the current density, $\Omega_{c}$ the conductive region and $r$ the distance between the integration point and the observation point. In our case, we consider a constitutive law linking the current density $\mathbf{J}$ to the electric field $\mathbf{E}$ :

$$
\mathbf{J}=\sigma \mathbf{E}
$$

with $\sigma$ the conductivity of the conductive region. Equation 2 can be rewritten as follows:

$$
\frac{\mathbf{J}}{\sigma}=-j \omega \frac{\mu_{0}}{4 \pi} \int_{\Omega_{c}} \frac{\mathbf{J}}{r} d \Omega_{c}-\nabla V
$$

This equation is the governed equation of the PEEC inductive formulation. The current density $\mathbf{J}$ is interpolated by the first order face shape functions:

$$
\mathbf{J}=\sum \mathbf{w}_{i} I_{i}
$$

where $\mathbf{w}_{i}$ are the $i^{t h}$ face shape function and $I_{i}$ the current flowing through the face $i$. Equation 5 is discretized using the Galerkin method with face shape functions as projection functions. The obtained linear system is [11]:

$$
([R]+j \omega[L])\{I\}=\left\{U_{b}\right\}
$$

with

$$
\begin{aligned}
{[R]_{i, j} } & =\int_{\Omega_{c}} \frac{\mathbf{w}_{i} \mathbf{w}_{j}}{\sigma} d \Omega_{c} \\
{[L]_{i, j} } & =\frac{\mu_{0}}{4 \pi} \int_{\Omega_{c}} \mathbf{w}_{i} \int_{\Omega_{c}} \frac{\mathbf{w}_{j}}{r} d \Omega_{c} d \Omega_{c} \\
\left\{U_{b}\right\}_{i} & =-\int_{\Omega_{c}} \mathbf{w}_{i} \nabla V d \Omega_{c}
\end{aligned}
$$

Matrix $[R]$ is the resistive sparse matrix while $[L]$ is the full inductive matrix. This system leads to an RLM circuit presented in Figure 1

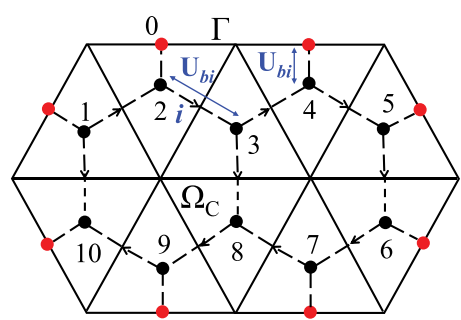

(a)

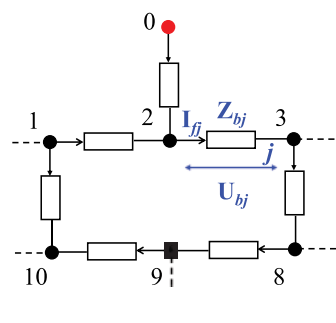

(b)
Fig. 1. (a) Primary and dual mesh (dashed line). The nodes and branches of the dual mesh are successively nodes and branches of the equivalent electrical circuit. (b) Part of the electrical circuit with $U_{b j}$ the voltage at the terminals and $Z_{b j}$ the equivalent impedance (self and mutual) of the branch $j$.

This circuit can be coupled with external lumped circuit elements and its final resolution is performed using independent nodes or independent loops analysis [23]. Let us notice that if we want to solve a problem with less restrictive assumptions including capacitive effects, the circuit can be completed with additional lumped capacitances. The following of the paper will present a strategy in order to do it in a economic way.

\section{EXTRACTION OF PARASITIC CAPACITIES}

The problem of extracting parasitic capacitances has a long history, in which the BEM approach is a widely used method and has proven to be very efficient because of its ability to handle complex geometries and infinite domains. There are two alternatives to this method : the direct formulation based on the integral boundary equation derived directly from Laplace equation [24] and the indirect formulation based on singlelayer and adjoint of double-layer potential equations (with dielectric regions) [25], [26], [27], [28], [29]. Several other developments have also been introduced such as BIM dual or pure second-kind BIM [30], [31], [32]. The Equivalent Charge Formulation (ECF) based on the indirect BEM formulation is chosen in this paper for its simplicity, its reliability and the possibility to be applied to dielectric multi-domains problems. 


\section{A. Equivalent Charge Formulation}

Let us consider a problem composed of several conductive regions $\Omega_{c i}$ with a given surface potential distribution and placed in a surrounding dielectric regions $\Omega_{d}$ like presented in Figure 2. The dielectric materials permittivity of region $\Omega_{d}$ is $\epsilon$. The permittivity of the free space is $\epsilon_{0}$. The surface $\Gamma_{c}$ denotes the union of the boundaries of $\Omega_{c i}$ regions and $\Gamma_{d}$ is the boundary between $\Omega_{d}$ region and free space. The boundary $\Gamma=\Gamma_{c} \cup \Gamma_{d}$ is the union of both boundaries where charges appear and has to be determined. Vector $\mathbf{n}$ denotes the external normal to $\Gamma_{d}$.

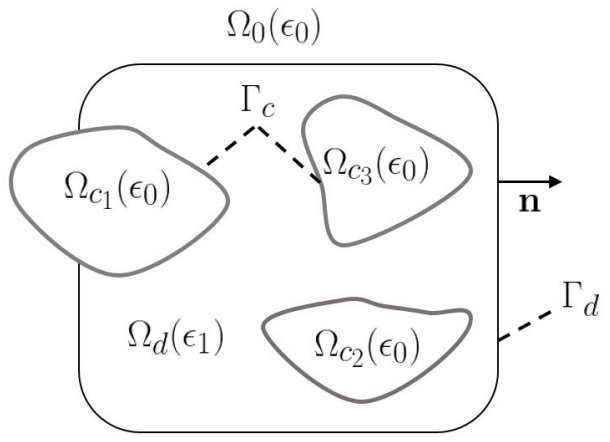

Fig. 2. Description of the 3D electrostatic problem that consists of one dielectric region and several volume conductive regions.

The equivalent charge density located on $\Gamma$ is $\rho_{s}$. This charge distribution has to be understood as the sum of free electrical charges and polarization charges. The single-layer potential created by this charge distribution at a target point $P$ belonging to the boundary of the conductive regions $\Gamma_{c}$ is written as:

$$
V(P)=\frac{1}{4 \pi \varepsilon_{0}} \int_{\Gamma} \frac{\rho_{s}}{r} d \Gamma
$$

A second integral equation based on the double-layer potential is obtained at point $P$ on $\Gamma_{d}$ by expressing the jump of the normal component of the electric field.

$$
0=-\frac{\left(\varepsilon+\varepsilon_{0}\right)}{2 \varepsilon_{0}\left(\varepsilon-\varepsilon_{0}\right)} \rho_{s}(P)+\frac{1}{4 \pi \varepsilon_{0}} \int_{\Gamma} \rho_{s} \nabla\left(\frac{1}{r}\right) \cdot \mathbf{n} d \Gamma
$$

By applying the Galerkin method with piece-wise constant shape functions to equations 11 and 12 projected on $\Gamma_{c}$ and $\Gamma_{d}$ respectively, we obtain the following system of equations:

$$
\left[\begin{array}{ccc}
P_{1,1} & \cdots & P_{1, n} \\
\vdots & & \vdots \\
P_{n_{c}, 1} & \cdots & P_{n_{c}, n} \\
E_{n_{c}+1,1} & \cdots & E_{n_{c}+1, n} \\
\vdots & & \vdots \\
E_{n_{c}, 1} & \cdots & E_{n, n}
\end{array}\right]\left\{\begin{array}{c}
q_{1} \\
\vdots \\
q_{n_{c}} \\
q_{n_{c}+1} \\
\vdots \\
q_{n}
\end{array}\right\}=\left\{\begin{array}{c}
V_{1} \\
\vdots \\
V_{n_{c}} \\
0 \\
\vdots \\
0
\end{array}\right\}
$$

where $q$ is the total charge per surface element, $n$ and $n_{c}$ are elements number of the discretized surfaces $\Gamma$ and $\Gamma_{d}$ respectively, $V_{i}$ is the imposed potential of element $i$ of the conductive regions. The expressions of matrices $P / E$ coefficients are :

$$
\begin{aligned}
& {[P]_{i, j}=\frac{1}{4 \pi \varepsilon_{0}} \int_{\Gamma_{c i}} \int_{\Gamma_{j}} \frac{1}{r} d \Gamma_{j} d \Gamma_{c i}} \\
& {[E]_{i, j}=\frac{1}{4 \pi \varepsilon_{0}} \int_{\Gamma_{d i}} \int_{\Gamma_{j}} \nabla\left(\frac{1}{r}\right) \cdot \mathbf{n} d \Gamma_{j} d \Gamma_{d i} \quad \text { if } i \neq j} \\
& {[E]_{i, i}=-\frac{\left(\varepsilon+\varepsilon_{0}\right)}{2 \varepsilon_{0}\left(\varepsilon-\varepsilon_{0}\right)} s_{i} \quad \text { if } i=j}
\end{aligned}
$$

where $s_{i}$ is the surface of element $i$. The linear system is solved with a multipole accelerated algorithm [25] coupled with a LU-MUMPS preconditioning technique. Once the charges $q$ have been obtained, we can compute the free electric charges $q_{e}$ per element which depend on the nature of the surface where they are located [33]. For the surface $\Gamma_{d}$, we have:

$$
q_{e}=q
$$

For an element belonging to interface $\Gamma_{c}$, we have:

$$
q_{e}=\varepsilon_{r} q
$$

with $\varepsilon_{r}$ the relative permittivity of the dielectric material.

Let us notice that if thin conductive regions are considered in the problem, the mesh can be reduced by only discretizing the averaged surface of the volume region. Moreover, multidielectric material regions problems can be efficiently treated with ECF formulation [28].

\section{B. Equivalent parasitic capacitances}

Let us consider a system of two conductors whose surfaces $\Gamma_{c 1}$ et $\Gamma_{c 2}$ are discretized by $n_{c 1}$ and $n_{c 2}$ elements. The parasitic capacitance system in the Kirchhoff sense is expressed in relation to electric charges $q_{e}$ and potentials $V$ of each surface element conductor according to the following equations:

$$
\begin{aligned}
q_{e 1} & =c_{1,1}\left(V_{1}-V_{r e f}\right)+\ldots+c_{1, i}\left(V_{1}-V_{i}\right)+\ldots+c_{1, n_{c}}\left(V_{1}-V_{n_{c}}\right) \\
& \vdots \\
q_{e i} & =c_{i, 1}\left(V_{i}-V_{1}\right)+\ldots+c_{i, i}\left(V_{i}-V_{r e f}\right)+\ldots+c_{i, n_{c}}\left(V_{i}-V_{n_{c}}\right) \\
& \vdots \\
q_{e n_{c}} & =c_{n_{c}, 1}\left(V_{n_{c}}-V_{1}\right)+\ldots+c_{n_{c}, i}\left(V_{n_{c}}-V_{i}\right)+\ldots+c_{n_{c}, n_{c}}\left(V_{n_{c}}-V_{r e f}\right)
\end{aligned}
$$

with $n_{c}=n_{c_{1}}+n_{c_{2}}, V_{i}$ the potential on element $i$ and $V_{r e f}$ the reference potential at infinity assumed to be $0 \mathrm{~V}$. Let us notice that the parasitic capacitance is a distributed parameter. However, if equipotential surfaces can be predicted on the conductive regions, lumped capacitances can be associated to these surfaces, leading to a good approximation of the distributed effect. Assuming that each surface of two conductors is considered as an equipotential surface, the model of the capacitive coupling between them is presented in Figure 3

In such case, equivalent capacities are determined by imposing uniform potentials on conductors and then by solving 13 


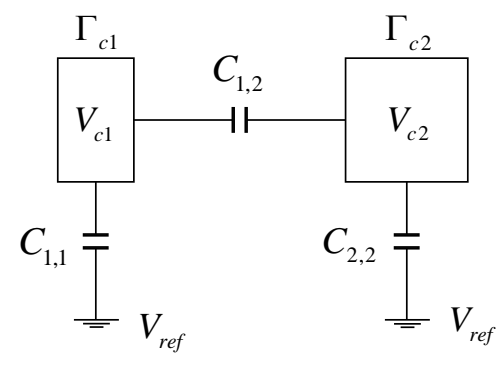

Fig. 3. Capacitive coupling for a system of two conductors with each of their surfaces considered as equipotential.

and computing the charges. Lumped capacitances are obtained with equations $19,20$.

$$
C_{1,1}=\sum_{i=1}^{n_{c 1}} q_{e i}, C_{1,2}=\sum_{i=n_{c 1}+1}^{n_{c}} q_{e i}
$$

with $V_{1}, \ldots, V_{n_{c 1}}=V_{c 1}=1 ; V_{n_{c 1}+1}, \ldots, V_{n_{c}}=V_{c 2}=0$

$$
C_{2,2}=\sum_{i=n_{c 1}+1}^{n_{c}} q_{e i}, C_{2,1}=\sum_{i=1}^{n_{c 1}} q_{e i}
$$

with $V_{1}, \ldots, V_{n_{c 1}}=V_{c 1}=0 ; V_{n_{c 1}+1}, \ldots, V_{n_{c}}=V_{c 2}=1$

$C_{1,2}$ and $C_{2,1}$ are capacitances between two same conductors. In other words, the capacitance matrix obtained from the above solution should be symmetrical. However, numerical errors may lead to a loose of this symmetry. One of the most important causes of this problem is an inadequate surface meshes of the regions [21]. For complex geometry problems, the implementation of an adaptive meshing technique can be useful [21].

\section{RLM-C COUPLING MODEL}

\section{A. Coupling principle}

In this part, we explain our coupling strategy. Figure 4 shows an example of two infinitely thin conductive plates supplied by a voltage source $V$ at one end and short-circuited at the other one. Assuming that both plates can be divided into four equipotential domains, we obtain a system of 8 equivalent parasitic capacitors which can be evaluated with the ECF formulation presented in the previous section. By combining these components with the RLM circuit obtained by inductive PEEC, we obtain a complete RLM-C coupling (see lower part of the Figure 47. In order to be able to neglect the propagative effect, it is necessary to check that the characteristic length of the problem geometry $D$ is smaller than $\lambda / 10$ where $\lambda$ is the wavelength.

In our approach, the determination of equipotential domains is an important task which cannot be done a priori except maybe by an expert user. We propose an alternative in order to make this determination automatic. The method starts with the analysis of the potential map obtained from the solution of the RLM inductive problem. The equipotential domains are determined and the overall RLM-C circuit is constructed and solved according to the aforementioned approach. After
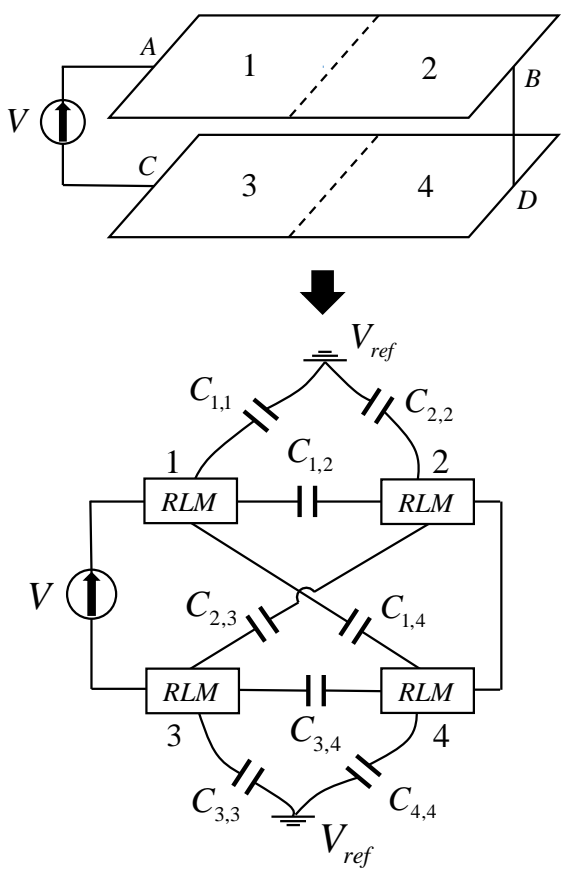

Fig. 4. Representation of a RLM-C coupling principle

solving the circuit, the potential map is updated again according to the new solution obtained. Such a process has to be repeated until the difference of the potential maps between two iterations is sufficiently small. However, we have found that at low frequencies, convergence occurs immediately because the potential map changes very little. In other words, it means that the capacitive effect is only a disturbance of the inductive effect and a strong coupling is not necessary in order to model the problem. Of course at higher frequencies, inductive and capacitive effects are more strongly coupled so iterations are needed to find a stabilized potential map. If dielectric domains are added, the connection model remains the same and only the capacitance values vary according to the free electrical charges calculations. In order to be able to create the previous coupled model, two tasks need to be done automatically : the equipotential domains determination and the localization of the connection points to the RLM circuit for capacitances. These will be presented in the following paragraph.

\section{B. Determination of equipotential regions}

As mentioned previously, the equipotential domains can be defined manually during mesh construction, like proposed in [19]. Another way to proceed is to use the potential distribution map obtained from the inductive PEEC problem to construct equipotential domains automatically. The potential of each surface element is the potential obtained at the corresponding dual node in the equivalent RLM circuit. Let us suppose we have computed a solution of the only-inductive problem and let us choose $n_{c l}$ the number of domains we want to consider as equipotential, we apply the following procedure:

1) Consider the potential values of all conductive surface elements, determine their maximum and minimum val- 
ues $\left(V_{\max }, V_{\min }\right)$ and compute the amplitude of an equipotential zone $\Delta V=\left(V_{\max }-V_{\min }\right) / n_{c l}$

2) Group the geometric elements whose potentials at the dual nodes belong to the same potential interval $\left[V_{\min }+n \Delta V, V_{\min }+(n+1) \Delta V\right]$

3) Split zones which do not have a geometric link in each group of elements obtained in the previous step.

Step 2 and 3 are based on the DBSCAN algorithm with some adaptations to our problem. DBSCAN (density-based spatial clustering of applications with noise) is a data clustering algorithm initially proposed in [34] which relies on the estimated density of clusters to perform partitioning. A recent application of this algorithm to electromagnetic problem is presented in [35]. DBSCAN can be applied to the set of surface element barycenters of our problem. An example of two elements clusters returned by this algorithm is shown on the right side of Figure 5. The first is associated to all the black points and the other one to all the gray points. However, this clustering technique only based on spatial distance could sometimes not ensure the connectivity of the elements set in a 3D problem. For instance, if two plates are very close to each other the standard algorithm doesn't detect that they belong to two nonconnected regions.
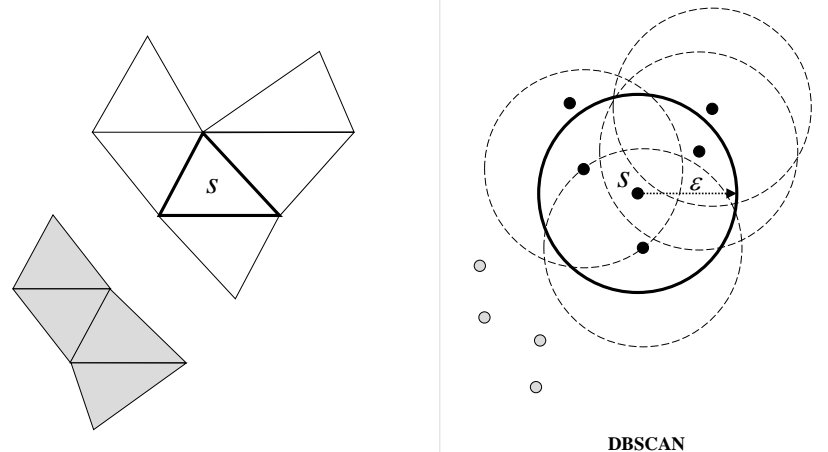

Fig. 5. Illustration of element clusters get with DBSCAN algorithm [34

For this reason we have tuned the algorithm in order to ensure the geometric connectivity of the elements in a cluster. In our version the neighbors of an element are found by analysing the edges it shares with others elements. The cluster extension algorithm then remains the same than the original DBSCAN algorithm.

The initial value for $n_{c l}$ is chosen a priory. Figure 6 shows the result obtained with our clustering algorithm on a meshed conductive plate fed by an external voltage source at $f=3 E 8 H z$ connected at both extremities. For a sake of simplicity, a $n_{c l}=4$ is chosen. The four clusters are clearly identified with good symmetry properties. Figure 4 presents results which would be obtained with two regions not geometrically related and with $n_{c l}=4$ as well.

\section{Determination of the connection points for lumped ca-} pacitances

For each cluster, the connection point of the lumped capacitance to the LRM circuit could be in principle chosen at any
A

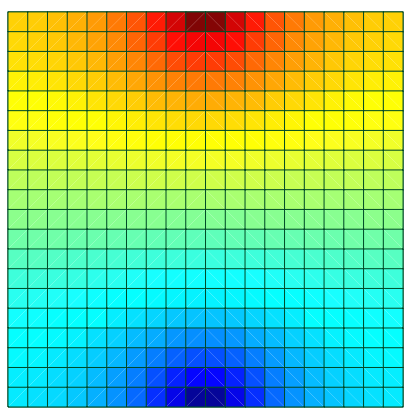

B
A

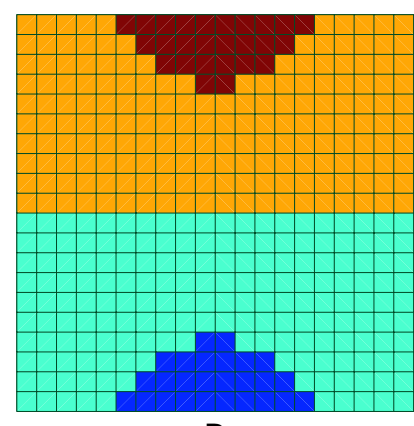

B

Fig. 6. The potential map (left) and four corresponding clusters on a plate at $f=3 \mathrm{E} 8 \mathrm{~Hz}$ (right).

point of the dual nodes of the domain elements since each region is considered as equipotential. From a theoretical point of view, the choice of the collecting point of the capacitive currents should not affect the final solution. However, if the region is large (i.e. includes a large number of mesh elements) this choice may slightly affect the solution because the concept of equipotential is an assumption. It then seems reasonable to select a point (or possibly several) where the value of the real potential is as close as possible to its mean value. According to equipotential region dertermination algorithm, we propose to choose as connection point the elements located in the geometrical center of each cluster (furthest to the border).

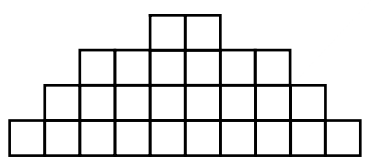

(a)

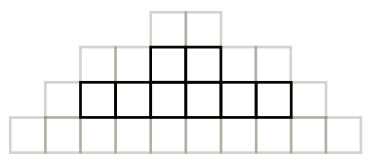

(b)

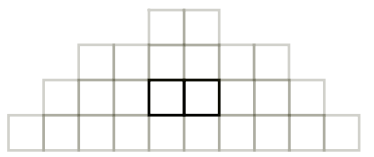

(c)

Fig. 7. Illustration of mesh reduction technique to find the connection point for capacitances

Each cluster is reduced by excluding the boundary elements leading to the elements with the highest neighbor density. This step continues until the cluster can no longer be reduced. This method depends heavily on the mesh and in the last step, not only one but a few admissible elements can be found. The procedure is illustrated in Figure 7. In this case, a single element is chosen for each adjacent set of remaining elements. Another example is shown in the Figure 8 In this example, two elements of the green cluster are found using the presented strategy. The dark blue elements on the figure are the obtained connection points and the value of the lumped capacitances are divided by the number of remaining elements in each cluster. 


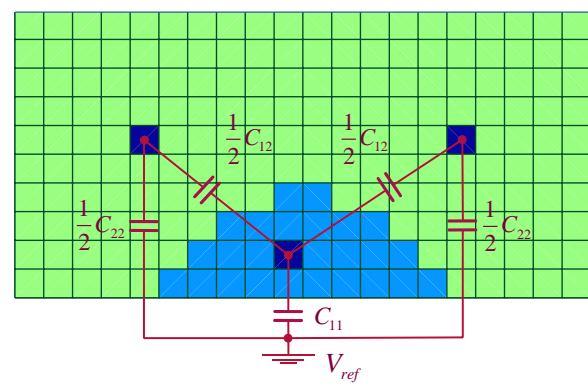

Fig. 8. An example of capacity coupling for two clusters

\section{ALGORITHM PRINCIPLE FOR FINAL RESOLUTION}

\section{A. Single frequency problem}

We consider first a problem with a single frequency. The RLM problem is solved. A first clustering is performed and lumped capacitances are computed and connected to the RLM circuit. The RLM-C problem is solved. A new potential map is obtained which leads to a new distribution of equipotential regions so a new set of lumped capacitances. The algorithm stops when the potential map is stabilized. In practice, the convergence is obtained after one or two iterations. The solving steps are presented in the diagram of Figure 9.

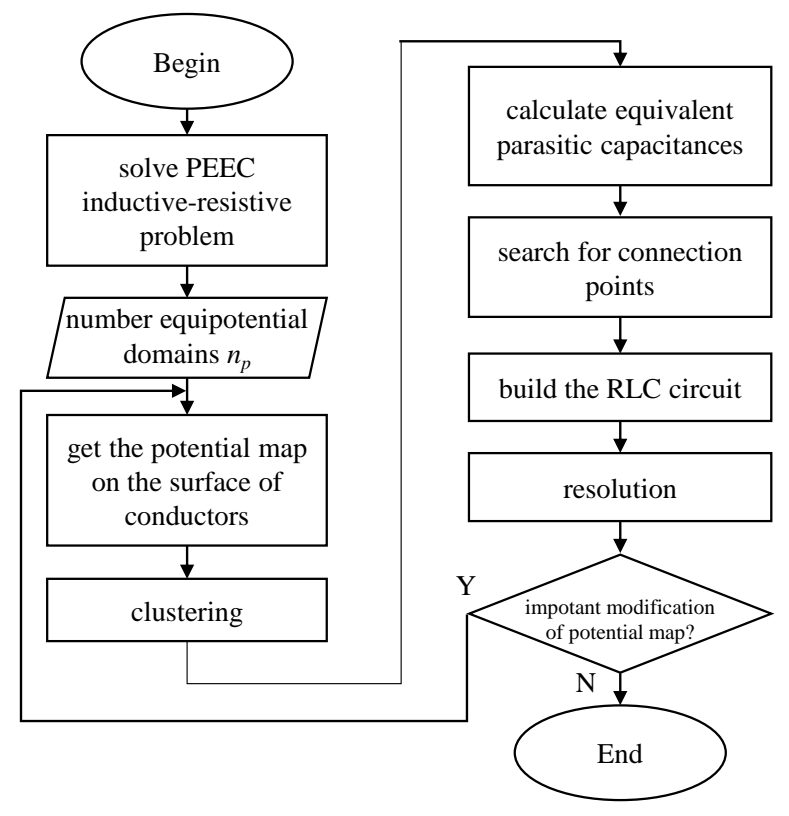

Fig. 9. Algorithm diagram for single frequency.

\section{B. Multifrequency problem}

If a problem with several frequencies is addressed, the previous algorithm has to be applied one time per frequency because the conductor potential map obtained by the solving of the inductive problem is frequency dependant. It means that a new RLM-C circuit topology is therefore necessary for each frequency. However, the generation and the resolution of a totally new equivalent electric circuit is time-consuming and in order to speed-up the computation time, we propose to start at the lowest frequency $f_{0}$ of the frequency range. The solving of the following frequency point is initialised with the previous clusters configuration and the capacitance computation process is adapted if needed. This strategy enables to save computation time. To start from the highest frequency would be another option but in the case of an high frequency, the algorithm would lead to an huge number of clusters for all the frequency range. In such a way, its advantage would be lost in term of computation time. The multifrequency algorithm principle is presented in Figure 10.

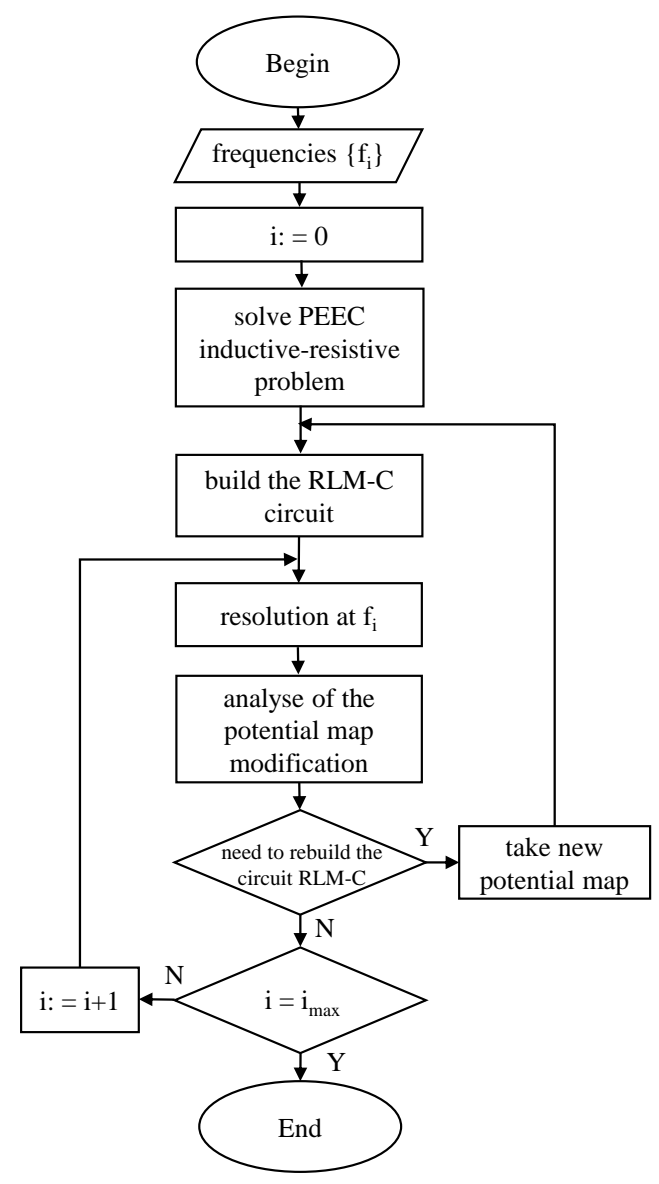

Fig. 10. Algorithm diagram for multi-frequency problem

\section{VALIDATIONS}

In this section, we propose some validations of the developed multifrequency algorithm presented in Figure 10 Three test cases are proposed with a growing complexity. Performances are compared to classical unstructured PEEC formulation presented in [13] where lumped capacitances are associated with each element of the mesh and not to equipotential domains. All the computation times for our approach include the full solving process i.e. the iteration procedure to obtain the potential map, the determination of equipotential domain and the connection points and all linear resolutions. All problems have converged after two iterations. 


\section{A. Test case 1}

A first example is proposed in order to proove the capability of the approach to catch an high capacitive effect which occurs even at low frequency. We consider a simple capacitance composed of two parallel square copper plates $50 \mathrm{~mm} \times 50 \mathrm{~mm} \times 35 \mu \mathrm{m}$. Both plates are placed at $1.6 \mathrm{~mm}$ from each other and connected to an external circuit composed of a voltage source of $U=1 \mathrm{~V}$, a resistance of $R=10^{4} \Omega$ and an inductance of $L=16 H$ in series. These values have been chosen in order to get a resonance frequency around $10 \mathrm{kHz}$ for the RLC circuit. The connection points to the external circuit are located in the middle of the plates (red square in Figure 11). The frequency varies from $9.7 \mathrm{kHz}$ to $10.5 \mathrm{kHz}$. The mesh is composed of 3,434 triangles and 5,446 circuit branches. The circuit analysis leads to the generation of 2,017 independent loops and a matrix system involving a total of 2,469 degrees of freedom. The RLM circuit topology obtained by the algorithm is open since there no electric contact between the two plates so each plate is considered as an equipotential region. Two clusters and associated connecting points which are located in the middle of the red zones are efficiently found by the algorithm.

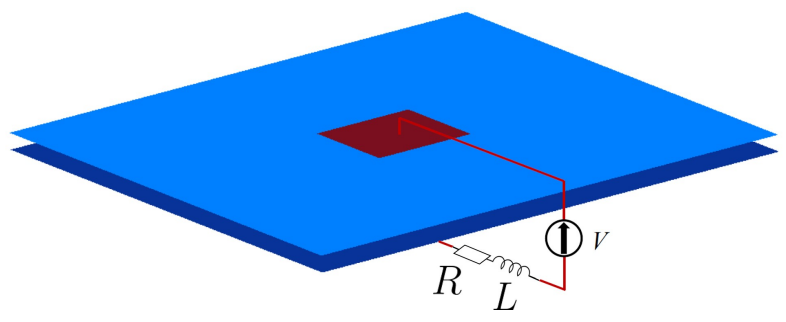

Fig. 11. Simple test case composed of two parallel conductive plates connected to a external voltage source, a resistance and an inductance.

Figure 12 shows the computed impedance obtained by our algorithm in comparison with the reference computed with thin shell unstructured PEEC formulation presented in [13]. The times needed for the clustering and the equivalent capacitances computation are $0.4 \mathrm{~s}$ and $12.6 \mathrm{~s}$ respectively. The total resolution time (for 41 frequencies) of our method is $95 \mathrm{~s}$, much shorter than the $1122 \mathrm{~s}$ needed for the classical formulation.

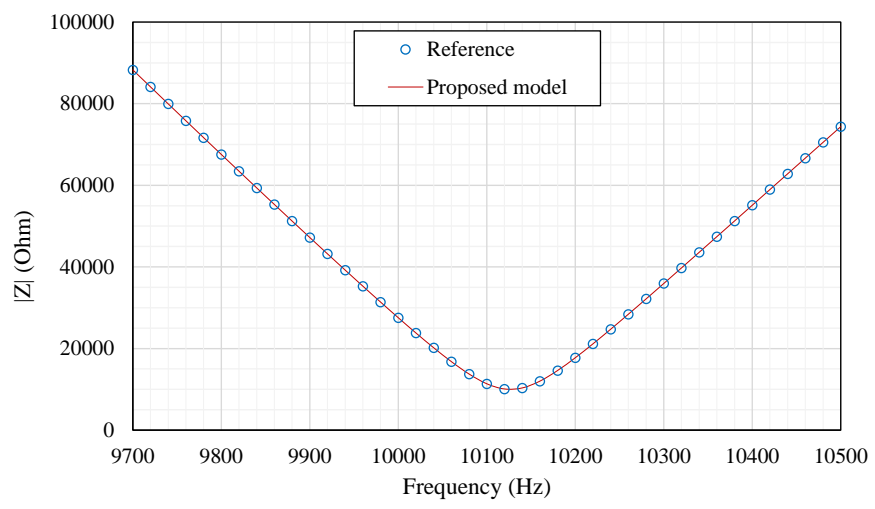

Fig. 12. Impedance magnitude curves obtained for test case 1 .

\section{B. Test case 2}

Let us now consider a more complex device consisting of two $35 \mu \mathrm{m}$ thick copper conductive surfaces separated by a $1.47 \mathrm{~mm}$ dielectric layer $\left(\epsilon_{r}=4.7\right)$ [21] The geometry is meshed with 1,794 quadrangles surface elements (2,338 circuit branches). The circuit analysis leads to 998 independent loops and 1,002 degrees of freedom for the matrix system. Both copper plates are short-circuited and the frequency range is from $1.0 E^{6} \mathrm{~Hz}$ to $2.0 E^{8} \mathrm{~Hz}$. The different obtained clusters $\left(n_{c l}=12\right.$ case $)$ are represented in the Figure 13 and are plotted in different colors. Connection points for lumped capacitances are also represented (deep blue elements).

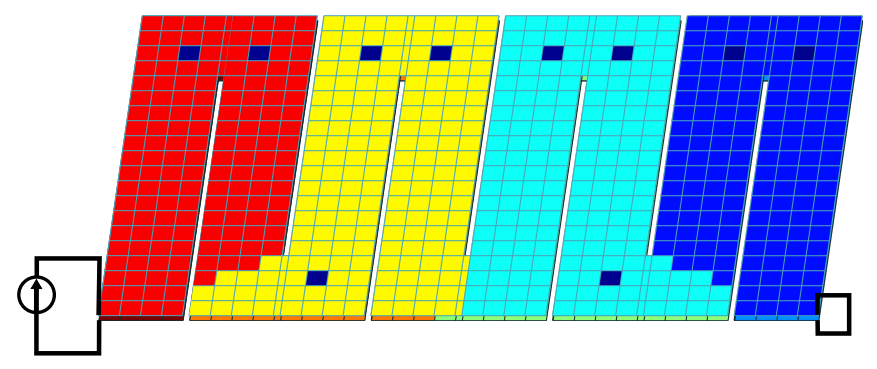

Fig. 13. Mesh of the serpentine in short-circuit mode with 8 clusters and their cores found at $f=1 E^{6} \mathrm{~Hz}$.

The numerical results are shown in Figure 14 The impedance curves are plotted with different clusters number $n_{c l}$. A system with very few equivalent parasitic capacitances like $n_{c l}=2$ can lead to results with poor accuracy from $1.2 E^{8} \mathrm{~Hz}$. When $n_{c l}$ is large enough, accuracy is much better and results are very similar to whose obtained with the complete formulation (see for instance $n_{c l}=24$ case). The black dashed line on the graph indicates the frequency $1.6 E^{8} \mathrm{~Hz}$ associated to $\lambda / D=10$ (i.e from this frequency the propagative effect can no longer be neglected). In order to analyse the convergence of our algorithm, the residual norms of GMRES at $f=1 E^{6} \mathrm{~Hz}$ (the capacitive effect is very low) and at $f=3 E^{7} \mathrm{~Hz}$ (the capacitive effect is quite stronger) with a relative stopping criterion $\epsilon=1 E^{-8}$ are presented in Figures 15, 16 and compared to the classical approach. Even if the numbers of degrees of freedom is not the same in both approaches, our method presents an excellent convergence rate of the linear solver demonstrating that the method significantly reduce the problems due to the poor conditioning of the complete formulation. Total resolution times are summarized in Table I For the $n_{c l}=24$ case, 8.5s and 49.s are needed for the clustering and the capacitances computation respectively. The total computation time is 320 s in this case.

TABLE I

SHORT-CIRCUIT SIMULATIONS TIME FOR 109 SAMPLING FREQUENCIES(TEST CASE 2)

\begin{tabular}{lcccc}
\hline \hline Test cases & $n_{c l}=2$ & $n_{c l}=8$ & $n_{c l}=24$ & Reference \\
\hline Resolution time (s) & 248.1 & 239.7 & 320.4 & 2344.9 \\
\hline \hline
\end{tabular}

Figure 17 shows the impedance magnitudes curve computed for the open-circuit case. Simulations were carried out with 


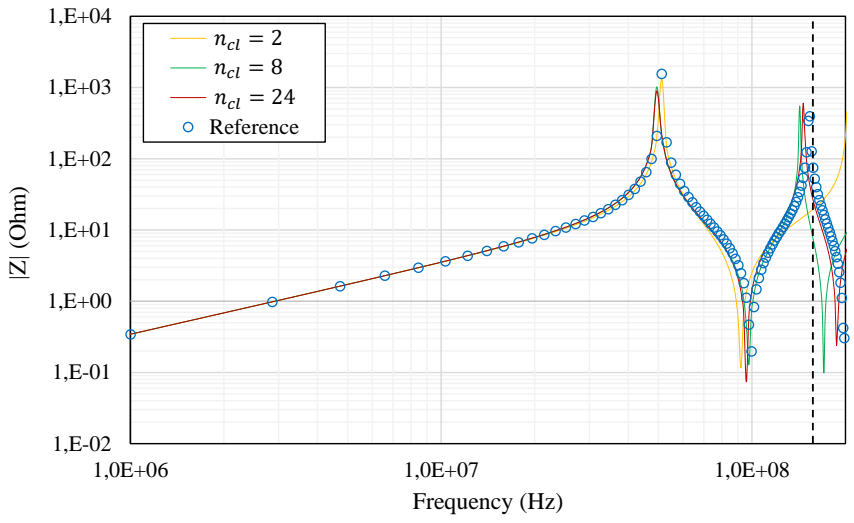

Fig. 14. Impedance magnitude curves for the test case 2 (short-circuit mode)

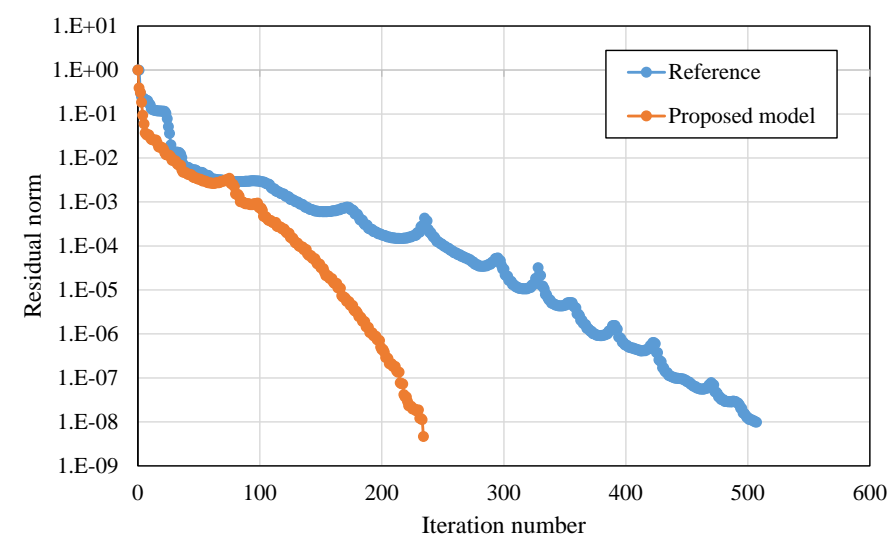

Fig. 15. Comparison of GMRES convergence rates at $f=1 E^{6} \mathrm{~Hz}$

the same mesh and with $n_{c l}=16$ clusters. RLM-C equivalent circuit is built at $23.11 \mathrm{Mhz}$. The results obtained and the reference results are very close, but the proposed model has gained a lot in terms of resolution time. With 109 sampling frequencies, we only need $293 s$ against $2516 s$ for the reference formulation.

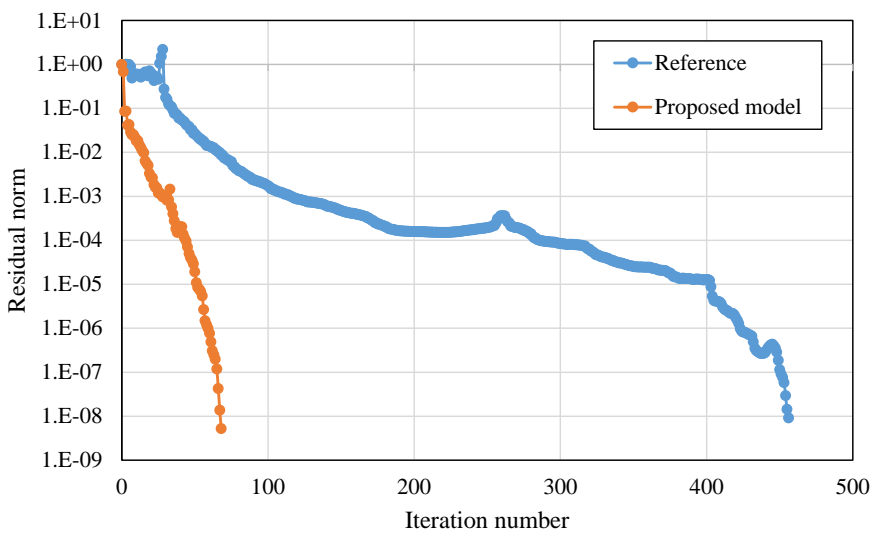

Fig. 16. Comparison of GMRES convergence rates at $f=3 E^{7} \mathrm{~Hz}$

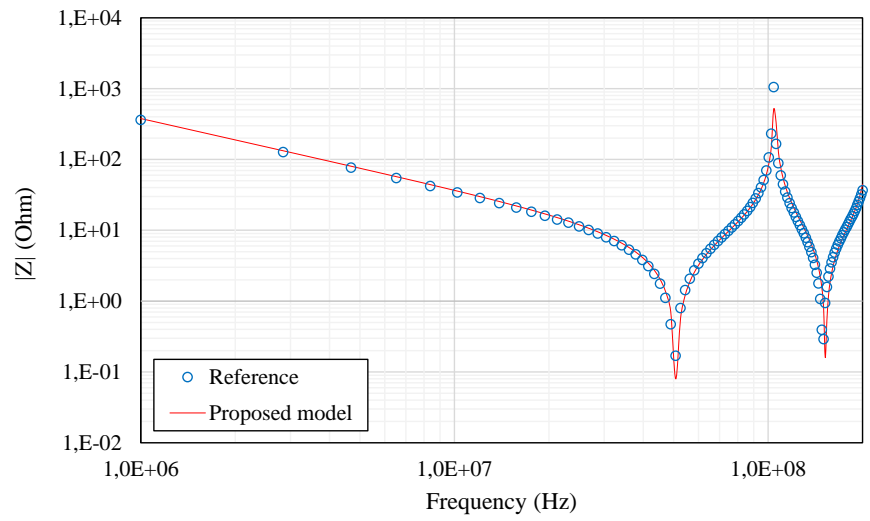

Fig. 17. Impedance magnitude curves for test case 2 (open-circuit mode)

\section{Test case 3}

Two previous examples have validated the coupling model with the use of the PEEC thin shell formulation [36]. We aim here to test it for the volume formulation. We consider a micro-coil [12] whose geometry is shown in Figure 18 In this example, the inductive-resistive circuit has been built using the PEEC volume formulation [11].

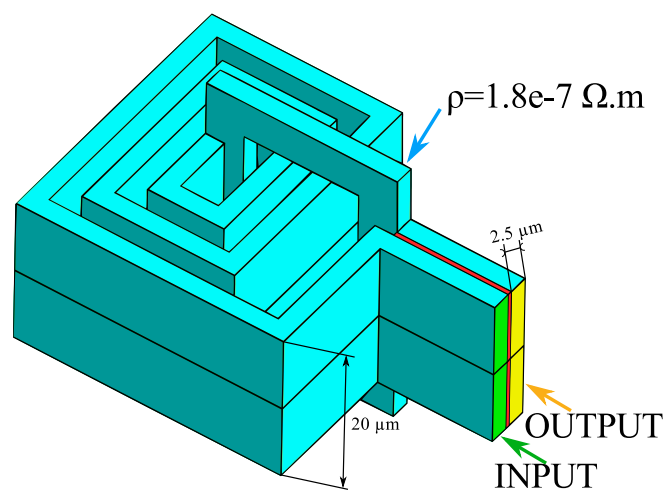

Fig. 18. Micro-coil with 3 spires [12]

The numerical results are shown in Figures 19 and 20 The mesh contains 4,822 tetrahedrons including 3,552 triangles on the surface associated to 7,451 circuit branches. The circuit analysis provide 2,838 independent loops and 2,871 degrees of freedom for the final matrix system. The number of obtained clusters in our test is $n_{c l}=12$. We obtained an excellent result close to the reference result. The resolution time for 41 sampling frequencies is $1156 \mathrm{~s}$ (clustering: $0.5 \mathrm{~s}$ and capacitances computation : 14.1s) while $4356 \mathrm{~s}$ are needed for the complete formulation.

\section{CONCLUSION}

This paper presents a new automatic strategy to build a complete RLM-C circuit based on the PEEC inductiveresistive circuit and on set of lumped capacitances calculated independently from an electrostatic integral formulation. The results obtained by the proposed method show a very good correlation with the complete PEEC method but the resolutions 


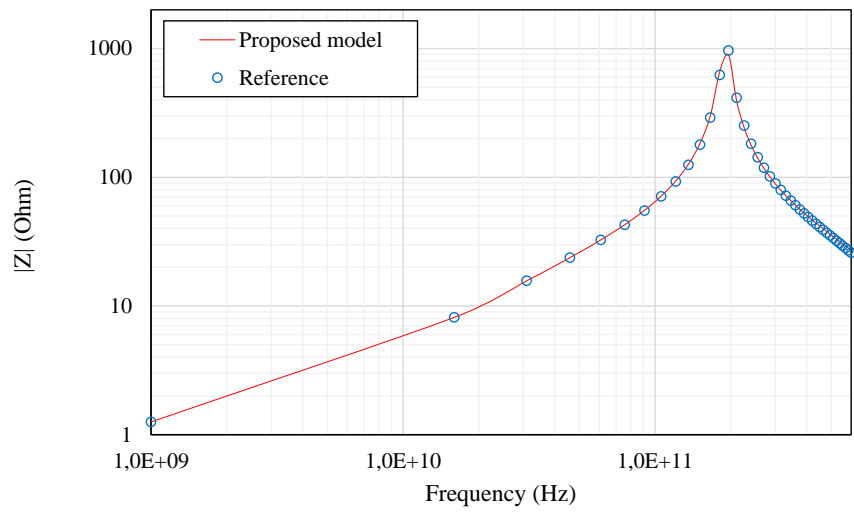

Fig. 19. Magnitude of the impedance depending on the frequency

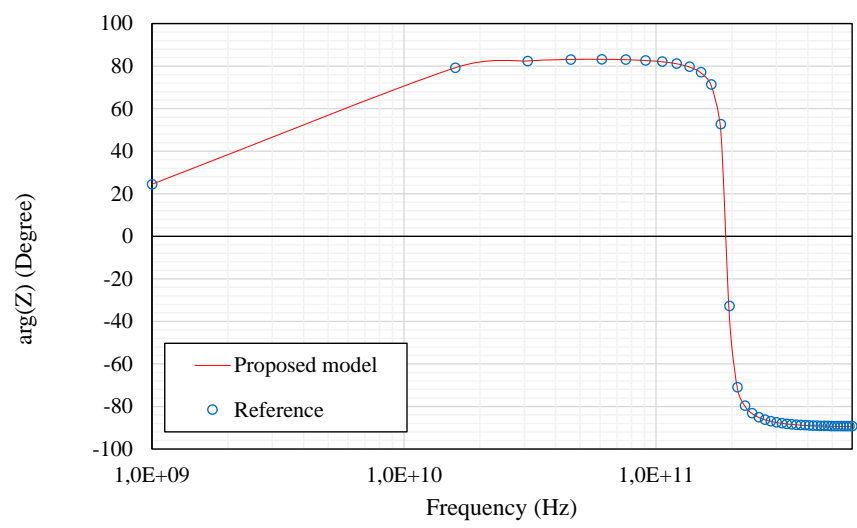

Fig. 20. Impedance phase depending on the frequency

converge much faster. Of course, this model is not applicable at high frequency (e.g. $\lambda / D<<10$ ) but is very interesting in the case of problem with important inductive and resistive effects but where capacitive effects cannot be neglected. As discussed in the paper, some additional work remains to be done to make this model more effective. The most important thing is developments for the construction of the mesh adapted to the electrostatic problem, especially with the presence of dielectric materials with high permittivity.

\section{REFERENCES}

[1] A. E. Ruehli, "Inductance calculations in a complex integrated circuit environment," IBM journal of research and development, vol. 16, no. 5 , pp. 470-481, 1972.

[2] A. Ruehli, G. Antonini, and L. Jiang, "Circuit oriented electromagnetic modeling using the peec techniques (wiley - ieee)," 072020.

[3] A. E. Ruehli, G. Antonini, J. Esch, J. Ekman, A. Mayo, and A. Orlandi, "Nonorthogonal peec formulation for time-and frequency-domain em and circuit modeling," IEEE Transactions on Electromagnetic Compatibility, vol. 45, no. 2, pp. 167-176, 2003.

[4] G. Antonini, "Peec capacitance extraction of 3-d interconnects," IET science, measurement \& technology, vol. 1, no. 4, pp. 201-209, 2007.

[5] A. E. Ruehli and H. Heeb, "Circuit models for three-dimensional geometries including dielectrics," IEEE Transactions on Microwave Theory and Techniques, vol. 40, no. 7, pp. 1507-1516, 1992.

[6] A. E. Ruehli and P. A. Brennan, "Capacitance models for integrated circuit metallization wires," IEEE Journal of solid-state circuits, vol. 10, no. 6, pp. 530-536, 1975.

[7] G. Antonini, A. Ruehli, and A. Haridass, "Peec equivalent circuits for dispersive dielectrics," in Proceedings of Piers-Progress in Electromagnetics Research Symposium, pp. 767-770, 2004.
[8] G. Antonini, M. Sabatini, and G. Miscione, "Peec modeling of linear magnetic materials," in Proc. IEEE Int. Symp. Electromagn. Compat., vol. 1, pp. 93-98, 2006.

[9] A. Bossavit, "Whitney forms: A class of finite elements for threedimensional computations in electromagnetism," IEE Proceedings A (Physical Science, Measurement and Instrumentation, Management and Education, Reviews), vol. 135, no. 8, pp. 493-500, 1988.

[10] F. Freschi, G. Gruosso, and M. Repetto, "Unstructured peec formulation by dual discretization," IEEE Microwave and Wireless Components Letters, vol. 16, no. 10, pp. 531-533, 2006.

[11] T. Nguyen, G. Meunier, J. Guichon, O. Chadebec, and T. Nguyen, "An integral formulation for the computation of 3-d eddy current using facet elements," IEEE Transactions on Magnetics, vol. 50, no. 2, pp. 549-552, 2014.

[12] J. Siau, Unstructured PEEC formulations considering resistive, inductive and capacitive effects for power electronics. Theses, Université Grenoble Alpes, Dec. 2016.

[13] J. Siau, G. Meunier, O. Chadebec, J. Guichon, and R. Perrin-Bit, "Volume integral formulation using face elements for electromagnetic problem considering conductors and dielectrics," IEEE Transactions on Electromagnetic Compatibility, vol. 58, no. 5, pp. 1587-1594, 2016.

[14] R. Torchio, P. Alotto, P. Bettini, D. Voltolina, and F. Moro, "A 3$\mathrm{d}$ peec formulation based on the cell method for full-wave analyses with conductive, dielectric, and magnetic media," IEEE Transactions on Magnetics, vol. 54, no. 3, pp. 1-4, 2017.

[15] G. Meunier, O. Chadebec, and J. Guichon, "A magnetic flux-electric current volume integral formulation based on facet elements for solving electromagnetic problems," IEEE Transactions on Magnetics, vol. 51, no. 3, pp. 1-4, 2015.

[16] G. Antonini and A. E. Ruehli, "Fast multipole and multifunction peec methods," IEEE Transactions on Mobile Computing, vol. 2, no. 4, pp. 288-298, 2003.

[17] G. Antonini and D. Romano, "Adaptive-cross-approximation-based acceleration of transient analysis of quasi-static partial element equivalent circuits," IET Microwaves, Antennas \& Propagation, vol. 9, no. 7, pp. 700-709, 2015.

[18] J. Siau, O. Chadebec, G. Meunier, J.-M. Guichon, and R. Perrin-Bit, "Preconditioning of a low-frequency electric field integral equation formulation with circuit coupling using h-matrices," in 2016 IEEE Conference on Electromagnetic Field Computation (CEFC), pp. 1-1, IEEE, 2016.

[19] V. Ardon, J. Aime, O. Chadebec, E. Clavel, and E. Vialardi, "Mom and peec method to reach a complete equivalent circuit of a static converter," in 2009 20th International Zurich Symposium on Electromagnetic Compatibility, pp. 273-276, 2009.

[20] V. Ardon, J. Aime, O. Chadebec, E. Clavel, J. Guichon, and E. Vialardi, "Emc modeling of an industrial variable speed drive with an adapted peec method," IEEE Transactions on Magnetics, vol. 46, no. 8, pp. 2892-2898, 2010.

[21] V. Ardon, Méthodes numériques et outils logiciels pour la prise en compte des effets capacitif dans la modélisation CEM de dispositifs d'électronique de puissance. Theses, Institut National Polytechnique de Grenoble - INPG, June 2010

[22] N. Bondarenko, T. Makharashvili, J. He, P. Berger, J. Drewniak, A. E. Ruehli, and D. G. Beetner, "Development of simple physics-based circuit macromodel from peec," IEEE Transactions on Electromagnetic Compatibility, vol. 58, no. 5, pp. 1485-1493, 2016.

[23] T.-S. Nguyen, J.-M. Guichon, O. Chadebec, G. Meunier, and B. Vincent, "An independent loops search algorithm for solving inductive peec large problems," Progress In Electromagnetics Research, vol. 23, pp. 53-63, 2012.

[24] C. A. Brebbia and S. Walker, Boundary element techniques in engineering. Elsevier, 2016.

[25] K. Nabors, J. White, et al., "Multipole-accelerated capacitance extraction algorithms for 3-d structures with multiple dielectrics," IEEE Transactions on Circuits and Systems I Fundamental Theory and Applications, vol. 39, no. 11, pp. 946-954, 1992.

[26] J. Tausch and J. White, "Capacitance extraction of 3-d conductor systems in dielectric media with high-permittivity ratios," IEEE Transactions on Microwave Theory and Techniques, vol. 47, no. 1, pp. 18-26, 1999.

[27] X. Cai, K. Nabors, and J. White, "Efficient galerkin techniques for multipole-accelerated capacitance extraction of 3-d structures with multiple dielectrics," in Proceedings Sixteenth Conference on Advanced Research in VLSI, pp. 200-211, IEEE, 1995.

[28] P.-G. Martinsson, "Fast evaluation of electro-static interactions in multiphase dielectric media," Journal of Computational Physics, vol. 211, no. 1, pp. 289-299, 2006. 
[29] J. Xiao, J. Tausch, Y. Cao, and L. Wen, "Combined equivalent charge formulations and fast wavelet galerkin bem for 3-d electrostatic analysis," International journal for numerical methods in engineering, vol. 79, no. 6, pp. 753-772, 2009.

[30] J. Tausch and J. White, "Second-kind integral formulations of the capacitance problem," Advances in Computational Mathematics, vol. 9, no. $1-2$, pp. 217-232, 1998.

[31] J. Tausch, J. Wang, and J. White, "Improved integral formulations for fast 3-d method-of-moments solvers," IEEE Transactions on ComputerAided Design of Integrated Circuits and Systems, vol. 20, no. 12, pp. 1398-1405, 2001.

[32] Y. Liu and L. Shen, "A dual bie approach for large-scale modelling of 3-d electrostatic problems with the fast multipole boundary element method," International journal for numerical methods in engineering, vol. 71, no. 7, pp. 837-855, 2007.

[33] T. K. Sarkar and R. F. Harrington, "The electrostatic field of conducting bodies in multiple dielectric media," IEEE Transactions on Microwave Theory and Techniques, vol. 32, no. 11, pp. 1441-1448, 1984.

[34] M. Ester, H.-P. Kriegel, J. Sander, and X. Xu, "A density-based algorithm for discovering clusters in large spatial databases with noise," vol. 96, pp. 226-231, 011996.

[35] D. Tratkanov, P. Prokopev, A. Ospina Vargas, and A. Hubert, "Vers un nouveau concept pour la génération automatique de réseaux équivalents à partir de calculs de type éléments finis," in 3ème édition du Symposium de Génie Electrique SGE 2018, (Nancy, France), July 2018.

[36] T. Nguyen, G. Meunier, J. Guichon, and O. Chadebec, "3-d integral formulation using facet elements for thin conductive shells coupled with an external circuit," IEEE Transactions on Magnetics, vol. 51, no. 3, pp. $1-4,2015$. 